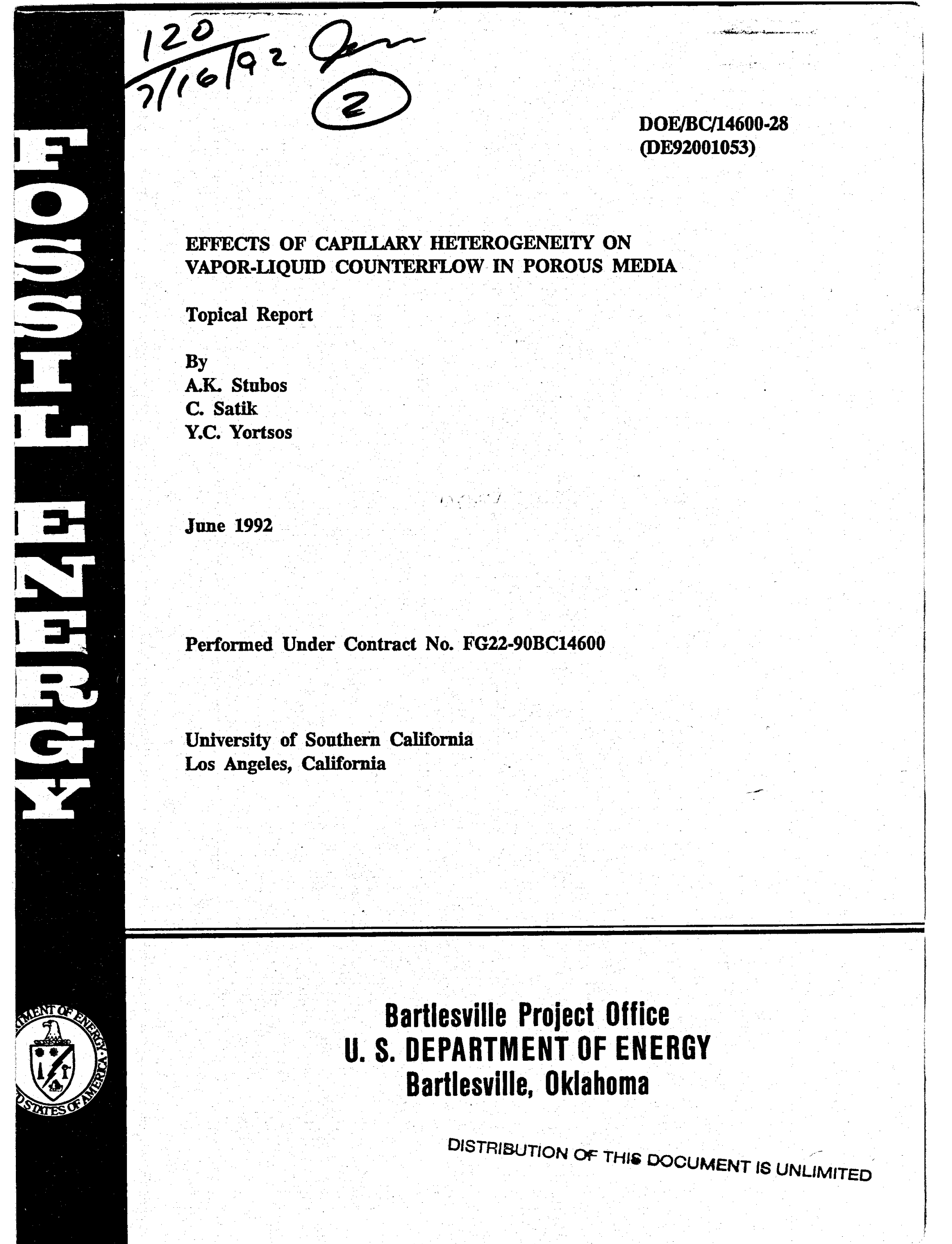




\section{DISCLAIMER}

This report was prepared as an account of work sponsored by an agency of the United States Government. Neither the United States Government nor any agency Thereof, nor any of their employees, makes any warranty, express or implied, or assumes any legal liability or responsibility for the accuracy, completeness, or usefulness of any information, apparatus, product, or process disclosed, or represents that its use would not infringe privately owned rights. Reference herein to any specific commercial product, process, or service by trade name, trademark, manufacturer, or otherwise does not necessarily constitute or imply its endorsement, recommendation, or favoring by the United States Government or any agency thereof. The views and opinions of authors expressed herein do not necessarily state or reflect those of the United States Government or any agency thereof. 


\section{DISCLAIMER}

Portions of this document may be illegible in electronic image products. Images are produced from the best available original document. 


\section{DISCLAIMER}

This report was prepared as an account of work sponsored by an agency of the United States Government. Neither the United States Government nor any agency thereof, nor any of their employees, makes any warranty, express or implied, or assumes any legal liability or responsibility for the accuracy, completeness, or usefulness of any information, apparatus, product, or process disclosed, or represents that its use would not infringe privately owned rights. Reference herein to any specific commercial product. process, or service by trade name, trademark, manutacturer, or otherwise does not necessarily constitute or imply its endorsement, recommendation, or favoring by the United States Government or any agency thereof. The views and opinions of authors expressed herein do not necessarily state or reflect those of the United States Government or any agency thereof.

This report has been reproduced directly from the best available copy.

Available to DOE and DOE contractors from the Office of Scientific and Technical Information, P.O. Box 62, Oak Ridge, TN 37831; prices available from (615)576-8401, FTS 626-8401.

Available to the public from the National Technical Information Service, U.S. Department of Commerce, 5285 Port Royal Rd., Springfield, VA 22161. 
DOE/BC/14600-28

Distribution Category UC-122

Effects of Capillary Heterogeneity on Vapor-Liquid

DOE/BC/14600--28

Counterflow in Porous Media

DE92 001053

Topical Report

By

A.K. Stubos

C. Satik

Y.C. Yortsos

June 1992

Work Performed Under Contract No. DE-FG22-90BC14600

Prepared for

U.S. Department of Energy

Assistant Secretary for Fossil Energy

T. B. Reid, Project Manager

Bartlesville Project Office

P. O. Box 1398

Bartlesville, OK 74005

Prepared by

University of Southern California

Department of Chemical Engineering

Petroleum Engineering Program

Los Angeles, CA 90089-1211 



\section{Contents}

List of Figures

Abstract $\quad 1$

1 Introduction 3

2 Formulation 4

A. Horizontal Counterflow $\ldots \ldots \ldots \ldots \ldots \ldots \ldots \ldots \ldots \ldots \ldots \ldots \ldots \ldots \ldots \ldots, \quad 5$

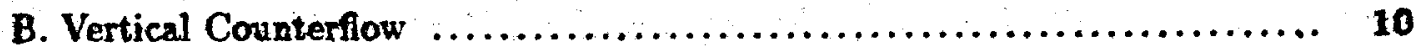

3 Conclusions 15

$\begin{array}{lll}\text { References } & 16\end{array}$ 


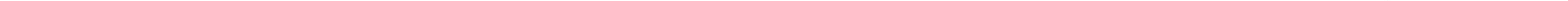




\section{List of Figures}

1 Schematic of counterflow geometry 18

2 Horizontal counterflow with $a>0$ : (a) $\tau$ (permeability) profile; (b) Saturation 19 response

3 The function $\omega(S)$ for capillary heterogeneity heat pipe (solid curve) and for 19 gravity heat pipe (dashed curve)

4 Saturation profiles for a $<$ : (a) $S_{1}<S_{V H}$; (b) $S_{V H}<S_{1}<S_{L H}$; (c) 20 $S_{L H}<S_{1}$; (d) $S_{L H}<S_{0}$; (e) $S_{V H}<S_{0}<S_{L H}$; (f) $S_{0}<S_{V H}$. Arrow denotes direction of integration

5 Steady-state selection in homogeneous heat pipes: (a) $S_{1}<S_{L G}$; (b) $S_{1}>S L G \quad 21$ ; (c) $S_{0}>S_{V G}$; (d) $S_{0}<S_{V G}$. Arrow denotes direction of integration

6 Normal heterogeneity: (a) $\tau$ profile; (b) $L D$ regime, $\omega=0.02$; (b) $V D$ regime, 22 $\omega=0.02$; (d) dimensionless capillary pressure for the LD regime

7 Transition to single phase flow for larger heat fluxes: (a) $L D$ regime, $\omega=0.2 ; 23$ (b) $V D$ regime, $\omega=0.2$

8 Weak heterogeneity: (a) $\tau$ profile; (b) $L D$ regime, $\omega=23.0$; (c) $V D$ regime, $\omega 24$ $=23.0 ;$ (d) $V D$ regime, $\omega=0.6$ 


\title{
EFFECTS OF CAPILLARY HETEROGENEITY ON VAPOR-LIQUID COUNTERFLOW IN POROUS MEDIA
}

\author{
A.K. Stubos, C. Satik and Y.C. Yortsos \\ Department of Chemical Engineering \\ Petroleum Engineering Program \\ University of Southern California \\ Los Angeles, California 90089-1211
}

\begin{abstract}
Based on a continuum description, the effect of capillary heterogeneity, induced by variation in permeability, on the steady state, countercurrent, vapor-liquid flow in porous media is analyzed. It is shown that the heterogeneity acts as a body force, that may enhance or diminish gravity effects on heat pipes. Selection rules that determine the steady states reached in homogeneous, gravity-driven heat pipes are also formulated. It is shown that the "infinite" two-phase zone may terminate by a substantial change in the permeability somewhere in the medium. The two possible sequences, liquid - liquid dominated - dry, or liquid - vapor dominated - dry find applications in geothermal systems. Finally, it is shown that although weak heterogeneity affects only gravity controlled flows, stronger variations in permeability can give rise to significant capillary effects.
\end{abstract}




\section{INTRODUCTION}

Countercurrent vapor-liquid flows in porous media has been the subject of many recent studies due to its relevance to geothermal processes, boiling, thermal methods for oil recovery and nuclear waste disposal $[1],[2],[3],[4],[5]$. Of particular interest are steady state heat pipes driven by gravity. Current theory dictates that in homogeneous systems an infinitely long two-phase zone of constant saturation develops if the heating rate is low enough (below a critical value). Two such states are predicted, one corresponding to low liquid saturation (vapor-dominated, VD) and one corresponding to high liquid saturation (liquid-dominated, LD). In a recent note [6], we conjectured that the particular solution is selected based on the past history of the system. For instance, in boiling (bottom heating) it is the LD branch that is followed. While, in condensation of a superheated vapor (top cooling), it is the VD branch that is selected [7]. In either case, however, capillarity is necessary to connect the constant saturation profiles to the subcooled or dry regions, respectively.

In practice, of course, all systems are finite and heterogeneous. The two-phase zone must terminate at a finite location, where the "infinite" extent results of the homogeneous case break down. Termination of the two-phase zone must be obtained by smoothly merging the two-phase region with either a subcooled liquid or a dry region, in the two cases respectively (otherwise, non-zero vapor and liquid fluxes would exist at the impermeable boundary [6]). It has been tacitly implied in previous studies that this can be accomplished with a sharp permeability change. Analogous considerations apply to the gravitational stability of vaporliquid counterflow, when a vapor-rich region underlies a subcooled liquid layer [8],[9]. The present consensus is that unconditional stability is possible only if a permability heterogeneity exists somewhere in the two-phase region.

Heat pipe instability in the much different context of the sensitivity of steady, 1D profiles to the boundary conditions, has also been considered. It was suggested [10] that, under certain boundary conditions, a VD solution is unconditionally "unstable" and must revert to a "stable" LD configuration, or vice versa, if the boundary conditions are reversed. While not immediately apparent, this problem is actually related to effects of heterogeneity. Indeed, for non-condensing fluid flows in porous media, boundary conditions can be successfully treated as effects of heterogeneity, where the change in permeability is abrupt and very large [11]. 
Analogous considerations apply for the countercurrent flow case, as discussed below.

Effects of heterogeneity on vapor-liquid concurrent flow were studied in [12], where a previous work on the steady state, two-phase flow of non-condensing fluids [11] was extended. Heterogeneity effects on countercurrent vapor-liquid flows, however, have not been systematically addressed (see also [13]) and they are currently poorly understood. As briefly described above, cases in point are the issues of the termination of the "infinite" two-phase zone, of the gravitational instability and of the sensitivity to boundary conditions. This paper aims at resolving some of these issues. Based on the key assumption that capillarity and permeability are interrelated, we theoretically investigate various effects of heterogeneity. We find that in 1-D vapor-liquid counterflow, permeability (capillary) heterogeneity acts much like a body force (e.g. gravity), with the additional property that it is spatially varying. Such heterogeneity may thus enhance or counterbalance gravity effects, depending on amplitude and variation.

The paper is organized as follows: We first consider the horizontal case, which allows for capillary effects only to be studied and for an exact solution to be developed. Then, we consider effects of gravity. Selection rules are developed for a homogeneous heat pipe when the heat flux is below critical. Next, we address heterogeneous gravity heat pipes at conditions of both slow and fast permeability variation. In all cases, heat conduction is neglected. The description is based on a continuum formalism and employs the concepts of equilibrium, saturation-dependent only, capillary pressure and relative permeability. Whether this formalism is adequate for rapidly. varying permeabilities is not questioned. It is conceivable that, at least in the case of sharp heterogeneities, some of the results may need further support, e.g. from more detailed pore network-level analysis.

\section{FORMULATION}

\section{Preliminaries}

The heterogeneous variable of interest to this work is permeability [11],[12]. As a result of the Leverett $J$-function representation, the variable mostly affected in the present $1 D$ connterflow is capillary pressure

$$
P_{c}=\frac{\sigma J(S)}{\sqrt{k}}
$$

Although the dimensionless function $J$ (as well as the relative permeabilities) may also be weakly 
varying with permeability, it is the dimensional $\sqrt{k}$-dependence that controls the capillary variation (see also [11] for a more detailed discussion). This decoupling of saturation and permeability on the capillary pressure is key to the present investigation.

In heat pipes, the importance of conduction is expressed through the dimensionless group [7] $K R_{m}=\frac{k L_{v}^{2} M_{w} P_{o} \rho_{v}}{\mu_{v} \lambda R T_{o}^{2}}$. Typically, $K R_{m}$ is large (equal to 5184 for the conditions in [3]) and conduction is negligible. Conduction must be retained, however, in systems with low $k$ or high $\lambda$ values, although such cases may be of limited practical interest (but see [6]). Conduction is also important in the geothermal systems of the type discussed by Schubert \& Straus [14], where its inclusion is necessary in order to sustain the counterflow. Regardless of the application, however, the relevance of conduction to a study of capillary heterogeneity should be small.

In its absence, saturation and temperature are decoupled from each other and the solution is obtained by simple means. Following [7], a straightforward manipulation of mass, momentum and energy balances yields the simple equation:

$$
\tau J^{\prime} \frac{d S}{d \xi}-J \frac{d \tau}{d \xi}=\omega \frac{\left(k_{r \ell}+\beta k_{r v}\right)}{k_{r \ell} k_{r v}}+\sin \theta \tau^{2}
$$

Here $\tau \equiv \sqrt{k / k^{*}}$ is the heterogeneity variable which is spatially varying, $k^{*}$ denotes a constant reference permeability and superscript ' indicates derivatives with respect to $S$. The notation follows [7] except for $\tau$, which here measures permeability rather than temperature. The dimensionless coordinate $\boldsymbol{\xi}$ increases such that liquid velocity is positive, and vapor velocity and heat flux are negative, while the dimensionless heat flux $\omega=|q| \mu_{v} / k^{*} L_{v} g \Delta \rho \rho_{v}$ is normalized with a reference permeability. In this notation, therefore, different permeability regions have the same value of $\omega$, but not the same critical values (see also below). The angle $\theta$ is measured with respect to the horizontal in a counterclockwise sense (Fig. 1).

Equation (2) must be generally solved numerically. Preliminary insight can be obtained by an analytical solution, which is possible for a special case in horizontal counterflow. This case also highlights important effects of capillary heterogeneity.

\section{A. Horizontal Counterflow}

In a horizontal system $(\theta=0)$ counterflow is driven by capillarity alone [5] and (2) yields:

$$
\tau J^{\prime} \frac{d S}{d \xi}=\omega \frac{\left(k_{r \ell}+\beta k_{r v}\right)}{k_{r \ell} k_{r v}}+J \frac{d \tau}{d \xi}
$$

It is instructive to compare (3) with the equation corresponding to a homogeneous gravity heat 
pipe $(\theta=3 \pi / 2, \tau=1)$, which reads

$$
J^{\prime} \frac{d S}{d \xi}=\omega \frac{\left(k_{r \ell}+\beta k_{r v}\right)}{k_{r \ell} k_{r v}}-1
$$

Then, it becomes clear that capillary heterogeneity and gravity (second terms on the RHS of (3) and (2)) play similar roles. To explore this similarity, we consider the special case of (3) where $\tau$ is piecewise linear (Figure 2)

$$
\tau=\left\{\begin{array}{lll}
1 & ; & \xi<0 \\
a \xi+1 & ; & 0<\xi<d \\
\tau_{+} & ; & d<\xi
\end{array}\right.
$$

where $\tau_{+} \equiv a d+1$. In the above, the spatial extent of the heterogeneity was denoted by $d>0$, while $a$ indicates the direction of change $(a>0$ for an increase, $a<0$ for a decrease of $\tau$ ).

In the homogeneous region, $\xi<0$ or $d<\xi$, the solution is a continuously decreasing saturation obtained from (3) by setting $\tau=1$. Inside the heterogeneity, $0<\xi<d$, the saturation satisfies

$$
\frac{k_{r \ell} k_{r v} J^{\prime} d S}{\omega\left(k_{r \ell}+\beta k_{r v}\right)+k_{r \ell} k_{r v} a J}=\frac{d \xi}{a \xi+1}
$$

which can be readily integrated. Because of qualitatively different responses, two different cases will be considered.

\section{1. $a>0$ (Figure 2a)}

Here, the permeability is increasing and we obtain the straightforward result:

$$
\int_{S_{0}}^{S} \frac{k_{r \ell} k_{r v} J^{\prime} d S}{\omega\left(k_{r \ell}+\beta k_{r v}\right)+k_{r \ell} k_{r v} a J}=\frac{1}{a} \ln (a \xi+1)
$$

where $S_{o}$ is the saturation at 0 . Because of $a>0$, the saturation decreases steadily also within the region of heterogeneity (Figure $2 \mathrm{~b}$ ). The downstream value $S_{1}$ satisfies:

$$
\int_{S_{o}}^{S_{1}} \frac{k_{r \ell} k_{r v} J^{\prime} d S}{\omega\left(k_{r \ell}+\beta k_{r v}\right)+k_{r \ell} k_{r v} a J}=\frac{1}{a} \ln \tau_{+}
$$

provided that a solution to the latter exists. This requires:

$$
\int_{0}^{1} \frac{k_{r \ell} k_{r v}\left(-J^{\prime}\right) d S}{\omega\left(k_{r \ell}+\beta k_{r v}\right)+k_{r \ell} k_{r v} a J} \geq \frac{1}{a} \ln \tau_{+}
$$

Otherwise, single-phase flow conditions may develop inside the region of heterogeneity. The particular saturation profile depends on the conditions imposed outside this region. If the location of the subcooled liquid boundary on the left or the dry boundary on the right is known, 
then integration occurs from left to right or vice-versa, and $S_{0}, S_{1}$ etc. can be determined sequentially. The analogy of this result homogeneous gravity heat pipes is evident. The corresponding problem is top heating $\left(\theta=\frac{\pi}{2}\right)$ with vapor at the top and liquid at the bottom. Continuously decreasing saturation profiles for this problem with features qualitatively similar to the middle portion of Fig. $2 b$ have been derived before in [3] and [7].

Of special interest is the case of a sharp discontinuity $(a \gg 1)$. Then, (8) yields:

$$
\frac{J\left(S_{1}\right)}{J\left(S_{0}\right)}=\tau_{+}=\sqrt{\frac{k_{1}}{k_{0}}}
$$

which is the condition of constant capillary pressure, implying a saturation jump across the discontinuity. This well known static (no flow) condition differs from the case of concurrent flow [11]. In the latter, a build-up of the wetting phase saturation is necessary before a high permeability region is entered.

$2 . a<0$

More interesting results arise in the case of a permeability decrease. Indeed, when $a<0$, the denominator in (6) may vanish, if $\omega$ is small enough. For this to occur, the following equation must admit a real solution:

$$
\omega=-a J \frac{k_{r \ell} k_{r v}}{k_{r \ell}+\beta k_{r v}}
$$

The $R H S$ of (11) is schematically plotted in Figure 3 for $a=-1$. We note that there exists a critical value,

$$
\omega_{c r, H}=(-a) \max _{S}\left(\frac{J k_{r \ell} k_{r v}}{k_{r \ell}+\beta k_{r v}}\right)
$$

above which a real solution to (11) does not exist. The maximum is equal to 0.7 , hence the critical value is proportional to the heterogeneity intensity $(-a)$. Equation (12) suggests the existence of a critical heat flux

$$
q_{c r, H}=0.7 \frac{\sigma L_{v} \rho_{v}}{\mu_{v}}\left(-\frac{d \sqrt{k}}{d x}\right)
$$

the value of which increases with sharper changes in permeability. It follows that the saturation profile depends on the relative value of $\omega$ :

(i) For $\omega>\omega_{c r, H}$, equation (11) has no solution. The effect of heterogeneity is identical to the previous $(a>0)$, the solution described as in the schematic of Figure $2 b$,

(ii) For $\omega<\omega_{c r, H}$, equation (11) has two roots, denoted by $S_{V H}$ and $S_{L H}\left(0<S_{V H}<S_{L H}<1\right)$, to one of which the solution is attracted. This is in very close analogy with the vapor-dominated 
or liquid-dominated regimes of homogeneous gravity heat pipes. In the latter, the two attractors $S_{V G}$ and $S_{L G}$ are solutions of the equation

$$
\omega=\frac{\tau^{2} k_{r \ell} k_{r v}}{k_{r \ell}+\beta k_{r v}}
$$

which gives rise to a critical value $\omega_{c r, G}$ (equal to 0.3063 [3] for $\tau=1$ (Fig.3)). The corresponding critical heat fiux is

$$
q_{c r, G}=0.3 \frac{L_{v} \rho_{v}}{\mu_{v}} g k \Delta \rho
$$

By a comparison of (15) with (13) it is evident that capillary heterogeneity $\sigma\left(\frac{d \sqrt{k}}{d x}\right)$ plays a role identical to gravity $g k \Delta \rho$. This similarity is further discussed later. As in gravity heat pipes, the saturation integral in (6) diverges at the two saturations $S_{V H}$ and $S_{L H}$, thus nearly flat saturation profiles (either VD or LD) develop to span the region of heterogeneity. Here, however, it is capillary heterogeneity, with the permeability decreasing in the direction of liquid flow and not gravity, that sustains the constant saturation profiles. The particular solution selected depends on the direction of integration as shown in the following.

Consider, first, integration from the vapor side. This requires that superheated vapor exists somewhere on the right so that we may integrate from the location $S=0$ in the negative $\xi$ direction. The saturation, $S_{1}$, reached at $\xi=d$, dictates how the solution behaves inside the heterogeneity:

- If $S_{1}<S_{V H}$, then $d S / d \xi<0$, and the saturation is rapidly attracted to the asymptotic value $S_{V H}$ (Figure $4 \mathrm{a}$ ). This is a vapor-dominated regime. Outside the heterogeneity, $\xi<0$, the integration is straightforward:

$$
\int_{S_{V H}}^{S} \frac{k_{r \ell} k_{r v} J^{\prime} d S}{\left(k_{r \ell}+\beta k_{r v}\right)}=\omega \xi
$$

This solution applies until conditions of subcooled liquid are reached $(S=1)$.

- If $S_{V H}<S_{1}<S_{L H}$, then $d S / d \xi>0$, and the saturation becomes again asymptotic to $S_{V H}$, except that the saturation is now decreasing in the short region before the asymptote is reached (Figure 4b).

- Finally, if $S_{L H}<S_{1}$, then $d S / d \xi<0$, but the saturation cannot be attracted to a flat profile. The latter does not develop, instead the saturation is described by the previous equations (7)-(9), much like case A.1 (Figure 4c).

Consider, next, integration from the liquid side. We assume that subcooled liquid exists somewhere on the left, such that we can proceed integrating from the location $S=1$ in the 
positive direction. If we denote by $S_{o}$ the saturation at $\xi=0$, the following options are possible: - If $S_{L H}<S_{o}$, then $d S / d \xi<0$, and the solution is attracted to the (liquid-dominated) value $S_{L H}$ (Figure 4d). After exiting the heterogeneity, further integration proceeds normally, much like in (16) until superheated vapor conditions are eventually reached $(S=0)$.

- If $S_{V H}<S_{o}<S_{L H}$, then $d S / d \xi>0$, and the solution is attracted to the same liquiddominated asymptote, except that now the saturation increases in the short region before this asymptote is reached (Figure $4 \mathrm{e}$ ).

- Finally, if $S_{o}<S_{V H}$, then $d S / d \xi<0$, but the solution is not attracted to a flat profile. Instead, it decreases relatively fast, much like the homogeneous case (Figure 4f).

Thus, depending on the direction of integration, two different solutions (a VD and an LD) emerge. This feature is particular to vapor-liquid counterflow. The selection was shown to be determined from the direction of integration, or equivalently from the past history of the system, which therefore attributes a large scale hysteresis [6]. The VD solutions of Figure 4 correspond to steady states reached by a system which is initially vapor-occupied and subsequently cooled from the left, while superheated conditions are maintained somewhere on the right. This is a condensation process (akin to imbibition). The LD solutions of Figure 4 correspond to steady states reached by an initially liquid-occupied system which is subsequently heated from the right, while subcooled conditions are maintained somewhere on the left. This corresponds to a boiling process (akin to drainage). We point out that hysteresis effects, but at the pore level, are routine in displacement processes. For the present case of vapor-liquid counterflow, however, the hysteresis (evaporation - condensation) also involves large scale aspects (indeed, in the present work $k_{r v}, k_{r \ell}$ and $J$ were taken invariant to direction).

Before we proceed further, it is worthwhile to consider the limit of a steep discontinuity $(a<0$ and $|a|>1)$. As readily confirmed, the two roots approach the respective limits $S_{V H} \rightarrow 0$ and $S_{L H} \rightarrow 1$ in this case. Because singularities are not encountered, one may formally take the limit of (8) at large $|a|$. The result is the previous condition of capillary pressure continuity (10), provided that the new saturation values do not lie close to either of the two extreme values, 0 or 1 . Otherwise, the term $\omega\left(k_{r \ell}+\beta k_{r v}\right) / k_{r \ell} k_{r v}$ can become comparable to $-a J$ and the saturation jumps do not correspond to capillary pressure continuity alone.

The above analysis also applies when the heterogeneity is slowly varying. As shown below, the solution is still a VD or LD branch, although of variable saturation values. When the $\tau$ 
- profiles are arbitrary, however, a numerical solution is necessary.

\section{B. Vertical Counterflow}

We consider, next, the case of vertical counterflow. Here, two generic configurations are possible, heating from the top $(\theta=\pi / 2, \sin \theta>0)$, and heating from the bottom $(\theta=3 \pi / 2, \sin \theta<0)$. Because it is more commonly encountered, we address the bottom heating case first.

\section{Bottom Heating}

Under this condition, equation (2) yields:

$$
\tau J^{\prime} \frac{d S}{d \xi}=\omega \frac{\left(k_{r \ell}+\beta k_{r v}\right)}{k_{r \ell} k_{r v}}-\left(\tau^{2}-J a\right)
$$

where $a(\xi)$ is the heterogeneity gradient, $a \equiv d \tau / d \xi$. Clearly, capillarity augments or diminishes ( $a<0$ or $a>0$, respectively) the effects of gravity (second term on the RHS of (17)). In the homogeneous case $(\tau \equiv 1, a \equiv 0)$, the $R H S$ above vanishes for the two saturation values $S_{V G}$ and $S_{L G}$ that solve equation (14), provided that $\omega<\omega_{c r, G} \approx 0.3$. We recall that a similar condition was also encountered in heterogeneous, horizontal counterflow. The selection mechanisms of the horizontal case are therefore very much appropriate for homogeneous, gravity driven heat pipes as well. This is considered below where we use similar arguments, now for a homogeneous medium. As in [10], a crucial role is played by the boundary conditions imposed.

\section{(i) Homogeneous Systems : Steady State Selection}

When the integration proceeds from the bottom (the "vapor side") upwards, it is the VD branch $S_{V G}$ which is selected, if the starting saturation $S_{1}$ lies to the left, $S_{1}<S_{L G}$ (Figure 5a). This would be the case if superheated vapor existed somewhere below, as in the bottom curve of Figure $5 \mathrm{a}$ (note also that because of $(10)$, any desired saturation value is possible as a starting point, see top curve of Figure 5a). In the interpretation of [6] this case could result from an initially superheated system that partly condenses due to top cooling. If $S_{1}>S_{L G}$, on the other hand, a flat profile does not develop and the saturation rapidly converges to $S=1$ (Figure $5 \mathrm{~b}$ ).

By contrast, when the integration proceeds from the top (the "liquid side") downwards, it is the LD branch, $S_{L G}$, which is selected, if the starting saturation $S_{0}$ lies to the right, $S_{o}>S_{V G}$ (Figure 5c). This is the case of subcooled liquid somewhere at the top, a typical application being boiling [6]. If $S_{0}<S_{V G}$, a flat profile does not develop, the saturation rapidly approaching the dry regime, $S=0$ (Figure $5 \mathrm{~d}$ ). We conclude that it is the past history of the system that determines the steady state solution. Evidently, all such saturation profiles are intrinsically stable. 
(ii) Sharp Discontinuity: Termination of an "Infinite" Two-Phase Zone

Consider next the case of an abrupt discontinuity $(|a| \gg 1)$. This analysis is necessary to explain how the VD or LD constant saturation profiles of heat pipes can merge with subcooled liquid or superheated vapor, respectively, thus how the theoretical "infinite" two-phase zone can terminate in practice.

Consider, first, integration from the bottom within a constant permeability region (such that $\left.\omega<\omega_{c r, G}\right)$. Then, a VD regime is rapidly reached. For a homogeneous medium, this regime is predicted to continue indefinitely (but see also [6] and [7]). Can this profile merge with another LD regime or with a region of subcooled liquid? The answer is negative to the first part, but not to the second. In either case, for a change in the regime the permeability must decrease to a lower value $k_{t}$ somewhere at the top. Because $a$ is positive and large, the response is as in the horizontal case and capillary pressure continuity (10) applies. If $k_{t}$ is such that $\omega$ remains below critical at the top (recall that the critical flux is proportional to $k$ ), the previous scenario (pertaining to Figures 5a-5b) applies and the solution is either another VD region or a rapid approach to subcooled liquid, depending on the particular conditions. If $\boldsymbol{\omega}>\boldsymbol{\omega}_{c, G}$ at the top, only a finite two-phase zone develops that rapidly ends by merging with a subcooled liquid region. From the previous analysis, quantitative estimates can be readily obtained.

If integration proceeds from the top, an LD region is rapidly approached, assuming $\omega<$ $\omega_{c r, G}$. For this flat profile to change, and for a dry region to be eventually encountered, two possibilities exist: If the bottom is at a low enough permeability, $k_{b}$, such that $\omega<\omega_{c r, G}$, the solution would rapidly approach dryout, after a short increase in the liquid saturation right after the discontinuity. Dryout may also be reached, however, if the bottom is at a higher permeability, such that the saturation jump across the discontinuity would result into low enough saturation values (smaller than $S_{V G}$ ). We can employ the previous scenario of Figure $5 \mathrm{~d}$ to infer that there will be a relatively fast approach to a superheated (dry) regime under such conditions.

In summary, for a gravity driven VD heat pipe to terminate, it is necessary that the permeability increases somewhere in the downwards direction. A VD region exists at the bottom, while subcooled liquid dominates the top. If the change of permeability is in the opposite direction, capillary pressure continuity cannot bring a qualitative change in the saturation state. In that case, the regime would always remain vapor-dominated. For a LD heat pipe, on the 
other hand, termination is possible by either a permeability decrease or a permeability increase, provided that they are both sufficiently high. Significantly, LD and VD branches never merge with each other, regardless of the heterogeneity. This contrasts some of the arguments of [10] in which an "unstable" VD regime becomes connected to a "stable" LD regime, and vice versa.

\section{(iii) General Heterogeneity Effects}

Consider, next, general heterogeneity effects with normal variations in $\tau$. Equation (17) suggests that heterogeneity enhances (makes more vapor-rich or liquid-rich) the respective VD or LD regimes when $a<0$, and acts to diminish them in the opposite case. In the numerical solution below we used $\tau$ profiles that satisfy a correlated fractional Brownian motion ( $\mathrm{fBm}$ ) in the spatial interval [0.1] with $H=0.8$, which appears to be the natural heterogeneity in many rocks [15]. Two cases were studied, a slow and a normal variation in heterogeneity. By a simple rescaling of the equations it can be shown that the first can be represented by a signal of the same variation as the second, except that the levels of $\tau$ must be higher.

Before we proceed, it is instructive to provide order of magnitude estimates on the importance of capillary heterogeneity relative to gravity. From either (2) or from (13) and (15) it is readily shown that gravity effects would predominate when the following is satisfied

$$
(-\Delta \sqrt{k} / \Delta x) \ll \Delta \rho g k / \sigma \equiv N_{B}
$$

where $N_{B}$ is the Bond number. For permeabilites in the order of 1 darcy $\left(10^{-8} \mathrm{~cm}^{2}\right)$ and for a steam-water system, the above condition requires permeability variations much smaller than 1 darcy $/ m$. Capillary heterogeneity is even more enhanced when permeabilities are lower, for example in the order of 1 mdarcy $\left(10^{-11} \mathrm{~cm}^{2}\right)$, where the above requires variations much smaller than $0.1 \mathrm{mdarcy} / \mathrm{m}$. It follows that in many practical cases, capillary effects will not be negligible, particulary when permeability decreases in the direction of liquid flow, where the gravity effects predicted by homogeneous heat pipes theory may be substantially altered.

Consider a normal variation of $r$ (Figure 6a). Here, the combination $\tau^{2}-a J$ changes sign often within the interval. The solution displays hysteresis again, depending on the direction of integration. Capillary effects are quite significant and a one-to-one correspondence with $r(\zeta)$ is not obeyed. In fact, for relatively steep increases in $\tau$, capillary pressure continuity may be in effect, as discussed above, resulting in lower saturation values. For an LD state this is contrary to common gravity effects. Consider, for instance, integration from the left, where an LD regime 
is obtained provided that $\omega$ is low enough (Figure $6 \mathrm{~b}$ ). As long as the $\tau$ variations are not too great, the saturation values are relatively constant (early part of Figure $6 \mathrm{~b}$ ). The saturation variation is mild even though regions of relative large increase in $\tau$ are traversed. This behavior is similar to the horizontal counterflow for a negative and large. At the point where a sharp increase is encountered and $a$ becomes large (around the mid-point of Figure 6a), capillarity dominates, capillary pressure continuity is enforced and the saturation falls significantly. If this decrease is not too large, a lower saturation state, but still of the VD type, will be followed in the remaining part.

Under the same conditions in $\omega$, a VD regime arises, when the integration is from the right (Figure 6c). The first part of the profile ( $\xi$ roughly between 0.5 and 1$)$ corresponds to heterogeneity with generally positive slope $(a>0)$, thus capillary pressure continuity applies, the saturation rising as lower permeabilities are encountered. The second part of the heterogeneity, however, involves a rather steep negative slope (between 0.15 and 0.4 ). After the saturation falls rapidly ( $\xi$ between 0.3 and 0.4 ), further large changes in permeability do not induce significant saturation response. This interpretation is supported by the variation of the capillary pressure (Figure 6d), where the regime of capillary pressure continuity at the right half of the interval is evident. When the heat flux increases, a transition to single phase region is possible, the LD or VD states reaching dryout (Figure 7a) or subcooled liquid (Figure 7b), respectively, in a short region. This occurs first near the location with the highest positive slope in $\tau$ (mid-point of the $\xi$ interval). We emphasize that no transition from an LD to a VD state or vice versa was noted, while the two regimes maintain their identity in regions of $\tau$ decreases, no matter how sharp the latter are.

Consider, next, a case of much slower variation, where gravity effects are likely to predominate. This case can also be analyzed asymptotically. We take $\tau \equiv \tau(\xi / \ell$, where $\ell \gg 1$, and rescale the spatial variable using $\ell$ as the characteristic length, $\xi \equiv \ell \zeta$, to obtain:

$$
\frac{1}{\ell}\left(\frac{k_{r \ell} k_{r v}}{k_{r \ell}+\beta k_{r v}}\right)\left(J^{\prime} \tau(\zeta) \frac{d S}{d \zeta}-J \frac{d \tau}{d \zeta}\right)=\omega-\frac{k_{r \ell} k_{r v} \tau^{2}(\zeta)}{k_{r \ell}+\beta k_{r v}}
$$

Since $\ell \gg 1$, the solution is the saturation $S(\zeta)$ that makes the RHS vanish, thus representing a gravity driven process in a weakly heterogeneous medium. This is similar to homogeneous heat pipes, except that, because $\boldsymbol{T}$ is now variable, there exists a continuum of curves similar to Figure 3, each for a fixed $r$ (or $\zeta$ ). Their intersection with the line of constant $\omega$ defines a continuum of $S(\tau)$ values, which when plotted in a $S(\zeta)$ diagram give the solution to the 
problem. As before, there are two possible branches, a VD and an LD, to which the solution is always attracted (much like the cases in [7] and [12]). Again, VD and LD sequences are followed closely without the branches ever becoming intertwined. It follows that given a direction of integration, there is a direct one-to-one correspondence between the heterogeneity $\tau$ and the saturation, $S$.

For a numerical example we used the profile of Figure 8a. Because the combination $\tau^{2}-a J$ is always positive, it is possible for the $R H S$ of (17) to vanish for all $\tau$ provided that $\omega$ is low enough ( $\omega<\omega_{c r, \min }$, where $\omega_{c r, \min }$ must be obtained numerically). According to (19), the solution must follow closely the variation of $\tau^{2}$, resulting into either an LD or a VD branch, depending on the direction of integration. Numerical results shown in Figures $8 \mathrm{~b}$ and $8 \mathrm{c}$ for the respective regimes verify the theoretical predictions. After a short interval, the profiles are attracted to the asymptotic states and, with a small spatial delay (of about 0.05), mimic the variation of $\tau^{2}$. The VD solution shows a weaker sensitivity due to the relatively narrower range of saturation values allowed. As predicted, saturations in the LD regime increase or decrease as $\tau$ increases or decreases, respectively, while the saturations in the VD regime follow opposite trends.

When the heat flux acquires larger values $\left(\omega>\omega_{c r, m i n}\right)$, there are spatial locations where the local critical values may be exceeded $\left(\omega>\omega_{c r}(\xi)\right)$. Then, the saturation departs from the corresponding regimes and becomes rapidly attracted to a single phase region (dryout in the case of an LD state or subcooled liquid in the case of a VD state). By contrast, for the very low values of $\omega$ typical of geothermal reservoirs, all saturation values in the VD regime are very low, hence the profile is very nearly flat (Figure $8 \mathrm{~d}$ ) despite the permeability variations. It is clear that the existence of a flat profile should not be taken to imply a homogeneous medium.

\section{Top Heating}

We close by briefly noting that similar results are obtained for the case of top heating $(\sin \theta=1)$. Now, equation (1) yields:

$$
\tau J^{\prime} \frac{d S}{d \xi}=\omega \frac{k_{r \ell}+\beta k_{r v}}{k_{r \ell} k_{r v}}+\left(\tau^{2}+J a\right)
$$

For $a>0$, then $d S / d \xi<0$ throughout, and the solution follows in a straightforward fashion. Likewise, a monotonic profile (quite similar to the horizontal case) is obtained for $a<0$ and sufficiently large $\omega$. If the latter is low enough, however, the heterogeneity may lead to 
gravity-like VD and LD regimes, much like in the horizontal case. The various subcases were exhaustively treated, and we shall not elaborate further.

\section{CONCLUSIONS}

Within the framework of a continuum description, effects of permeability heterogeneity on steady state, vapor-liquid counterflow in porous media were examined. Permeability variations affect two processes, gravity-driven flow and capillarity. The variations of the latter can be significant. It was shown that capillary heterogeneity acts like an external body force (such as gravity), with the additional property that it also varies spatially. A multiplicity of steady states similar to gravity-driven heat pipes was found for decreasing permeabilities in horizontal counterflow and for heat fluxes lower than a critical value. Vapor-dominated and liquid-dominated regimes were obtained using selection rules that were postulated to depend on the past history (transient state) of the system. The analysis was aided by an exact solution obtained for a special heterogeneity profile.

In retrospect, the analogy between capillary heterogeneity and gravity is not unexpected. In capillary-controlled displacements in pore networks, effects of either gravity or pore size heterogeneity can both be successfully described by the gradient percolation approach of [16]. In terms of continuum models, the analogy between capillary heterogeneity and gravity was noted in the concurrent flow study of [11]. In the latter case, however, the curve corresponding to $\omega(S)$ (which represented an augmented fractional flow curve) admits only one root, therefore there was stable attraction to a single root only. In [11], steady state saturation profiles were obtained by backwards integration starting from the outlet end (opposite to the flow direction), since the solution was ill-posed and rapidly diverged if integration started from the opposite end. In the present case of countercurrent flow, both sides can be used as starting points for the integration. As shown above, which end is taken is decisive on the selection of the particular solution. This was implicitly contained also in [10].

The selection rules were next applied to determine the steady state regimes in gravity-driven heat pipes in homogeneous systems. It was shown that the different regimes may never connect with each other, thus retaining their identity as long as the system remains in a two-phase state. The issue of the termination of the infinite two-phase zone was next analyzed. For VD systems 
it was shown that termination requires a sharp increase in the permeability (in the direction of increasing depth) somewhere in the medium. Across this discontinuity the underlying VD state is rapidly converted to a subcooled liquid. For LD systems on the other hand, dryout can be reached as depth increases by either a sharp increase or a sharp decrease of permeability. The emerging picture from top-to-bottom is thus, subcooled liquid - (discontinuity) - VD - dry region or subcooled liquid - LD - (discontinuity) - dry region, in the respective cases. This ordering may be helpful in the interpretation of geothermal systems.

The importance of capillary heterogeneity relative to gravity was demonstrated in a study of two different heterogeneity modes, a slow and a normal variation. For permeabilities that vary slowly, the main effect is due to gravity, and the saturation response may follow the permeability variation depending on heat flux values. Larger variations in permeability induce significant capillary effects. Often, capillary pressure continuity across a sharp permeability charge may lead to dryout or to subcooled liquid. Capillary pressure continuity is not necessarily the only appropriate condition relevant to a decrease in permeability. Such effects attribute a significant large scale hysteresis on the saturation profiles.

\section{References}

[1] J.M. Straus and G. Schubert. One dimensional model of vapor dominated geothermal systems. J. Geophysical Res., 86(B10):9433, 1981.

[2] A.K. Stubos and J-M. Buchlin. Boiling and dryout in unconsolidated porous media. in "Convective Heat and Mass Transfer in Porous Media", S. Kakac, B. Kilkis, F.A. Kulacki and F. Arinc Eds., NATO ASI Series E, Applied Sciences, Vol. 196, Kluwer Academic Publishers, 1991.

[3] K.S. Udell. Heat transfer in porous media considering phase change and capillarity - the heat pipe effect. Int. J. Heat Mass Transfer, 28:485, 1985.

[4] M. Prats. Thermal recovery. SPE Monograph, Vol. 7, Dallas, Texas (1982).

[5] C. Doughty and K. Pruess. A similarity solution for two-phase fluid and heat flow near high-level nuclear waste packages emplaced in porous media. Int. J. Heat Mass Thansfer, $33: 1205,1990$. 
[6] A.K. Stubos, C. Satik, and Y.C. Yortsos. Critical heat flux hysteresis in vapor-liquid counterflow in porous media. Int. J. Heat Mass Transfer, in press, 1992.

[7] C. Satik, M. Parlar, and Y.C. Yortsos. A study of steady-state steam-water counterflow in porous media. Int. J. Heat Mass Transfer, 34:1755-1771, 1991.

[8] G. Schubert and J.M. Straus. Gravitational stability of water over steam in vapordominated geothermal systems. J. Geophysical Res., 85(B11):6505, 1980.

[9] P.S. Ramesh and K.E. Torrance. Stability of boiling in porous media. Int. J. Heat Mass Transfer, 1990:1895-1908, 33.

[10] M.J. McGuinness, M. Blakeley, K. Pruess, and M.J. O'Sullivan. Geothermal heat pipe stability. Int. J. Heat Mass Transfer submitted, 1990.

[11] Y.C. Yortsos and J. Chang. Capillary effects on steady state flow in heterogeneous cores. Transport in Poraus Media, 5:399-420, 1990.

[12] M. Parlar, M. Zeybek, and Y.C. Yortsos. Steady state vapor-liquid concurrent flow: relative permeabilities and end effects. Paper SPE 20054, SPE California Regional Meeting, Ventura, CA, April 4-6, 1990.

[13] Y.K. Chuah and V.P. Carey. Analysis of boiling heat transfer and two phase flow in porous media with non-uniform porosity. Int. J. Heat Mass Transfer, 28:147-154, 1985.

[14] G. Schubert and J.M. Straus. Steam-water counterflow in porous media. J. Geophysical Res., 84:1621, 1979.

[15] B. Sapoval, M. Rosso, and J.F. Gouyet. The fractal nature of a diffusion front and the relation to percolation. J. Phys. (Paris) Lett., 46:L149-156, 1985.

[16] T.A. Hewett. Fractal distribution of reservoir heterogeneity and their influence on fluid transport. Paper SPE 15386, presented at the 61st Annual SPE Fall Meeting, New Orleans, LA, 1986. 


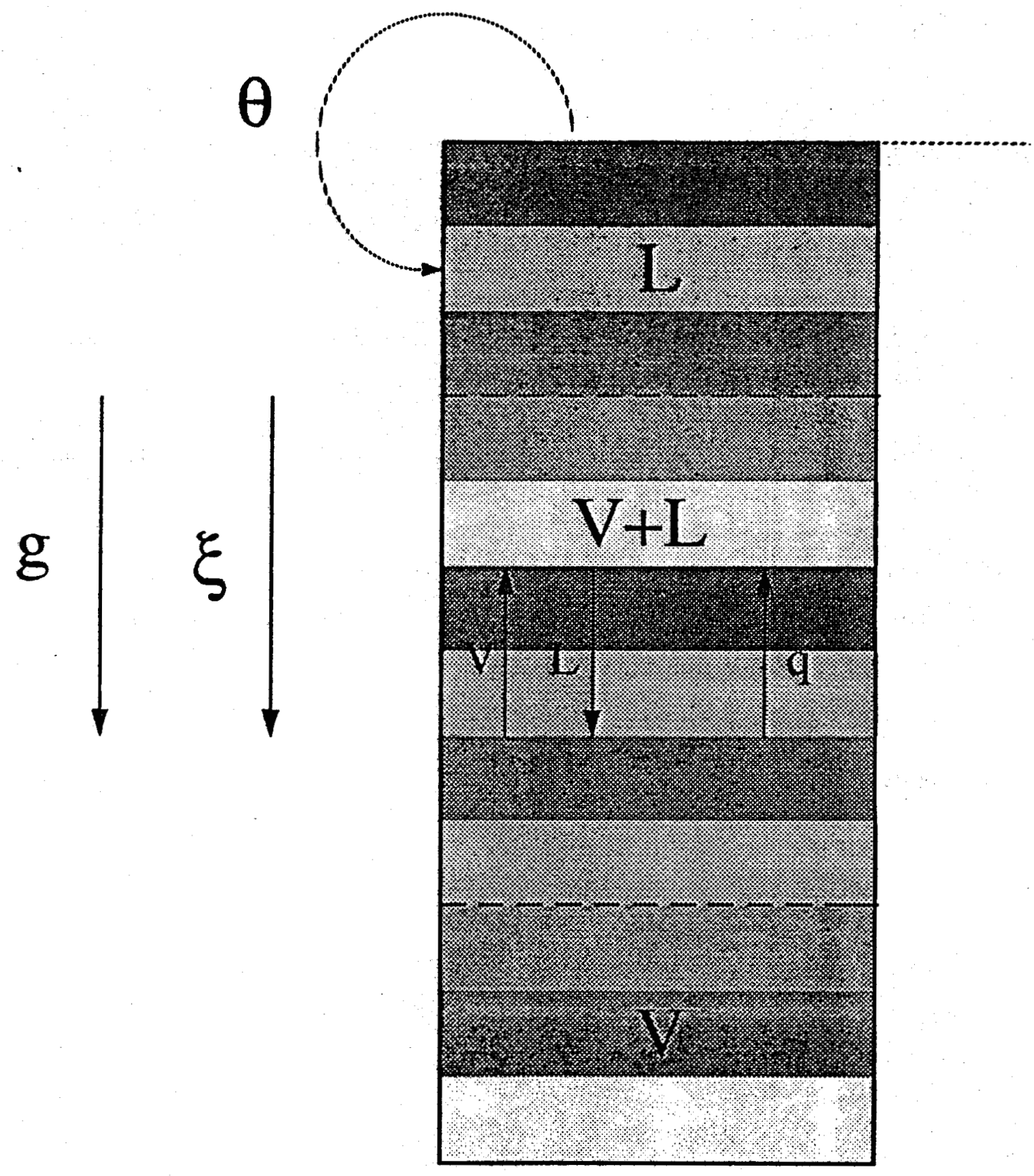

Figure 1. Schematic of counterflow geometry. 


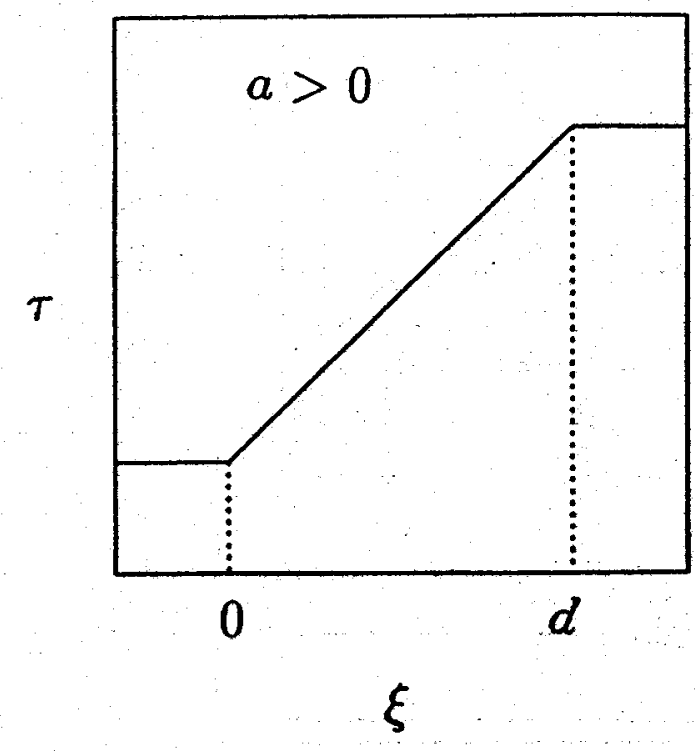

(a)

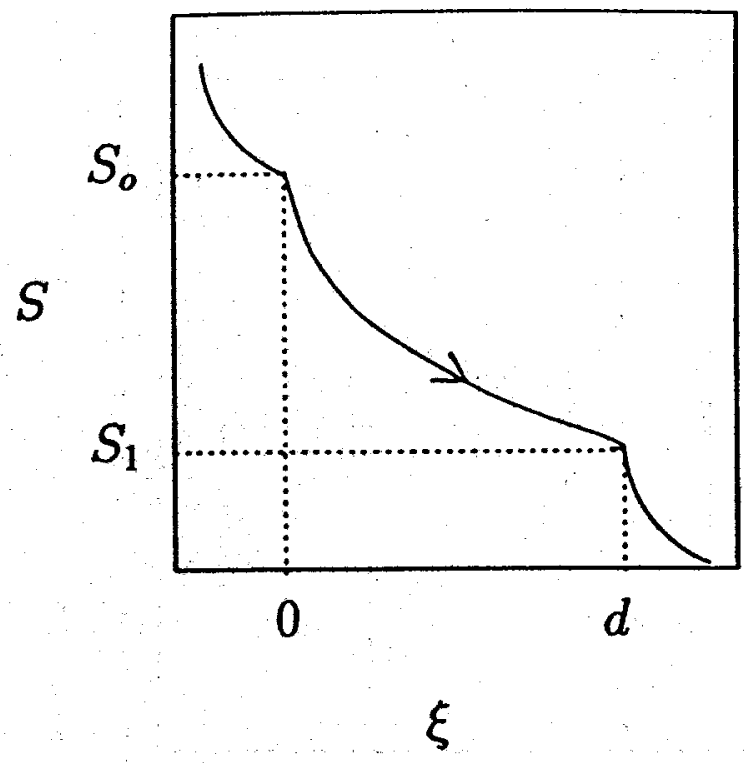

(b)

Figure 2. Horizontal counterflow with $a>0$ : (a) $\tau$ (permeability) profile; (b) Saturation response.

$\frac{J k_{r l} k_{r v}}{k_{r l}+\beta k_{r v}}$

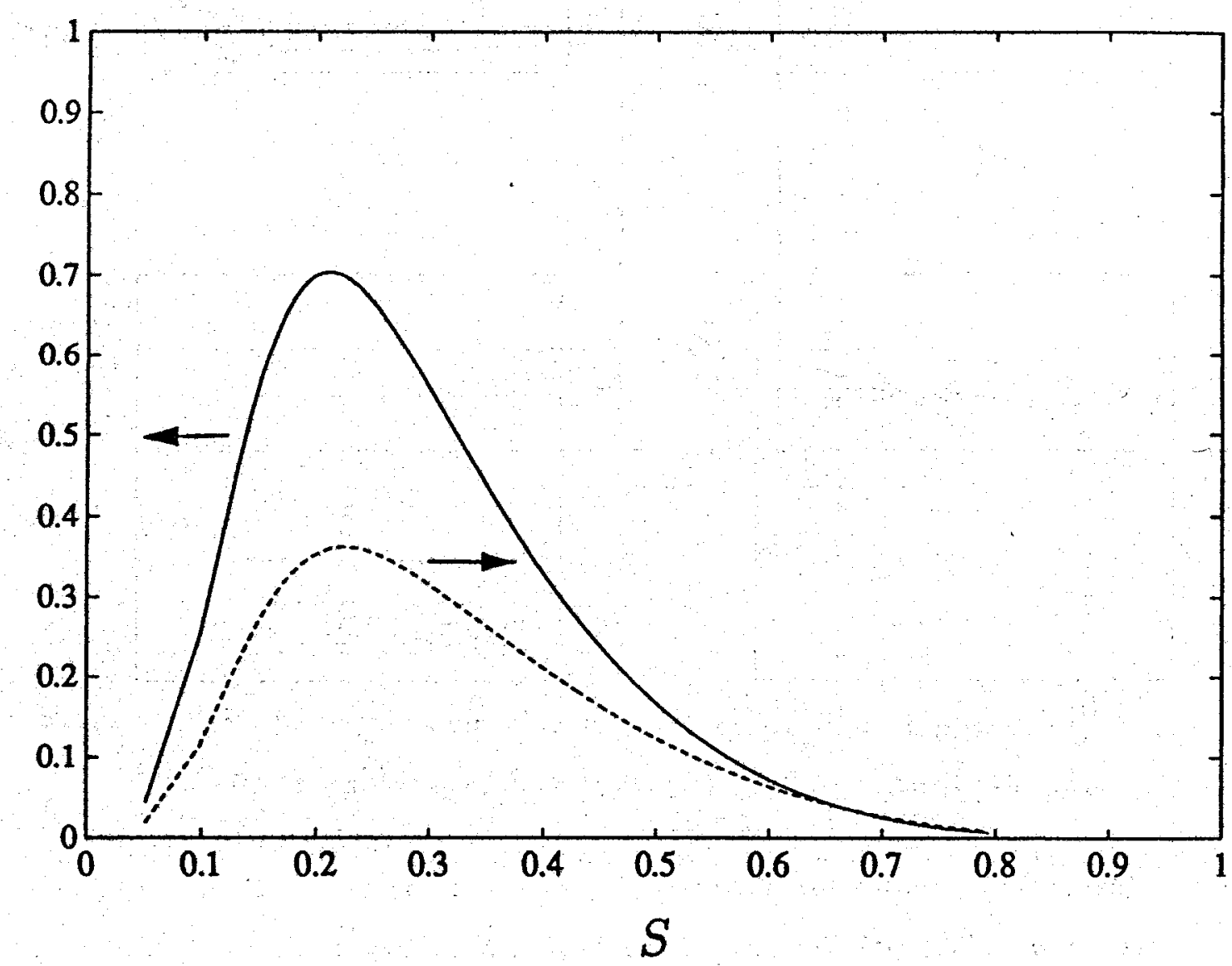

$\frac{k_{r l} k_{r v}}{k_{r l}+\beta k_{r v}}$

Figure 3. The function $\omega(S)$ for capillary heterogeneity heat pipe (solid curve) and for gravity heat pipe (dashed curve). 


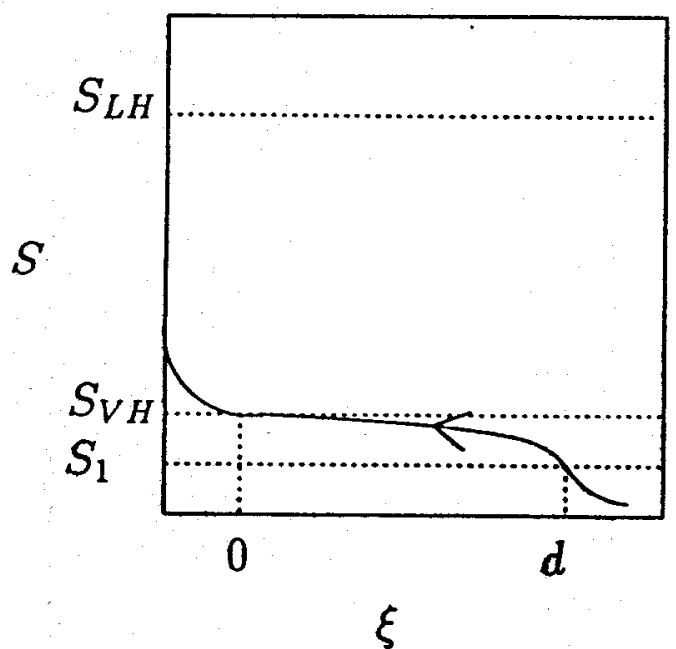

(a)

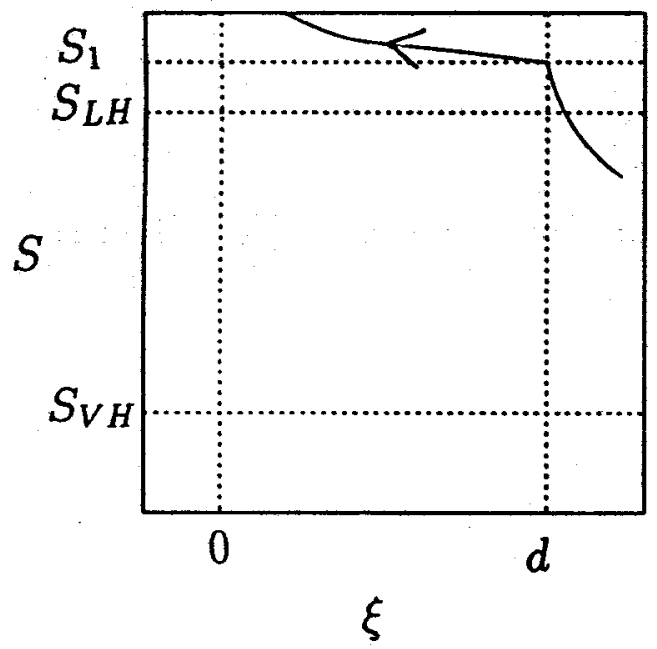

(c)

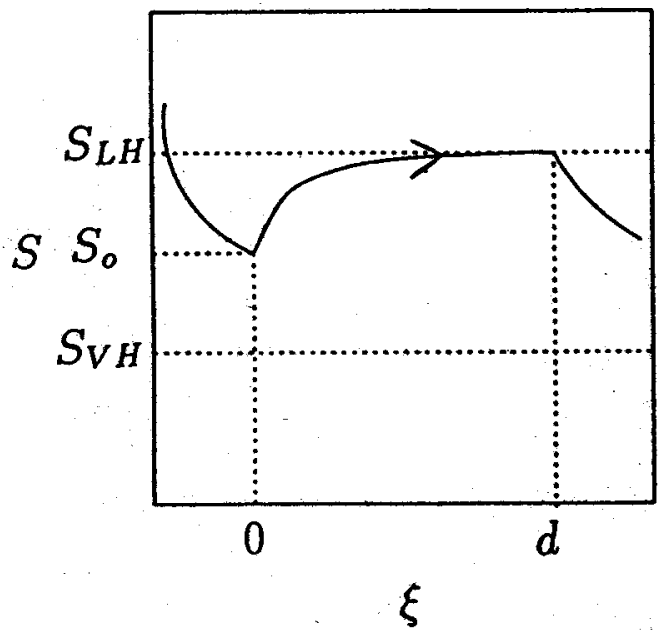

(e)

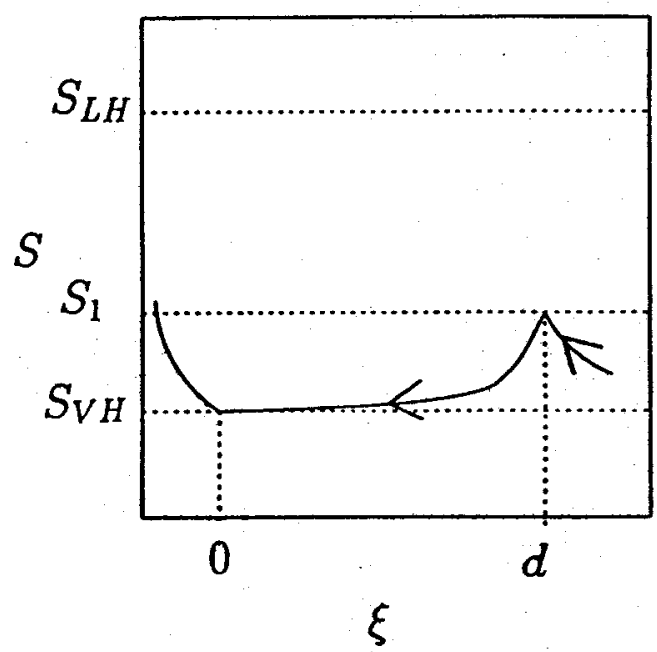

(b)

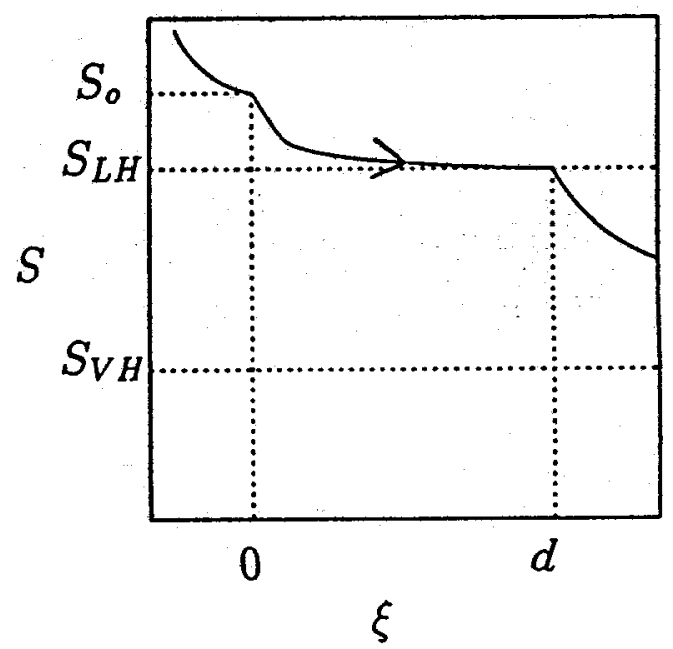

(d)

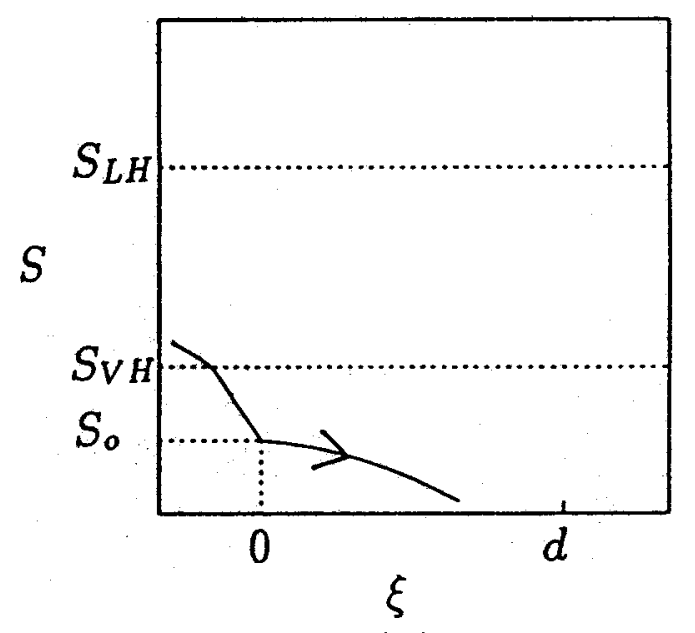

$(f)$

Figure 4. Saturation profiles for $a<0$ : (a) $S_{1}<S_{V H}$; (b) $S_{V H}<S_{1}<S_{L H}$; (c) $S_{L H}<S_{1}$; (d) $S_{L H}<S_{o}$; (e) $S_{V H}<S_{o}<S_{L H}$; (f) $S_{o}<S_{V H}$. Arrow denotes direction of integration. 


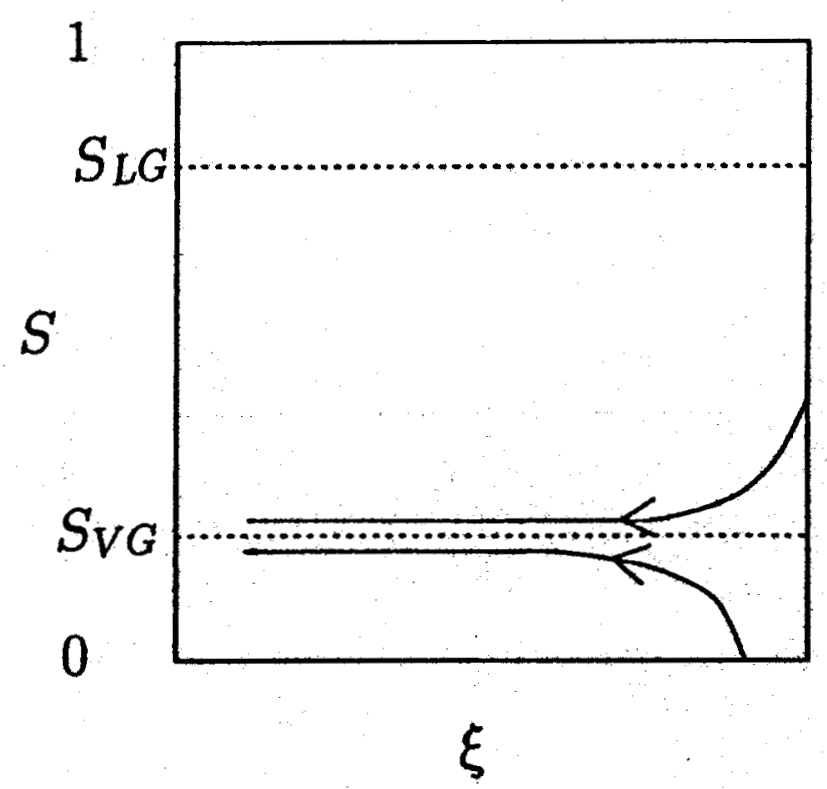

(a)

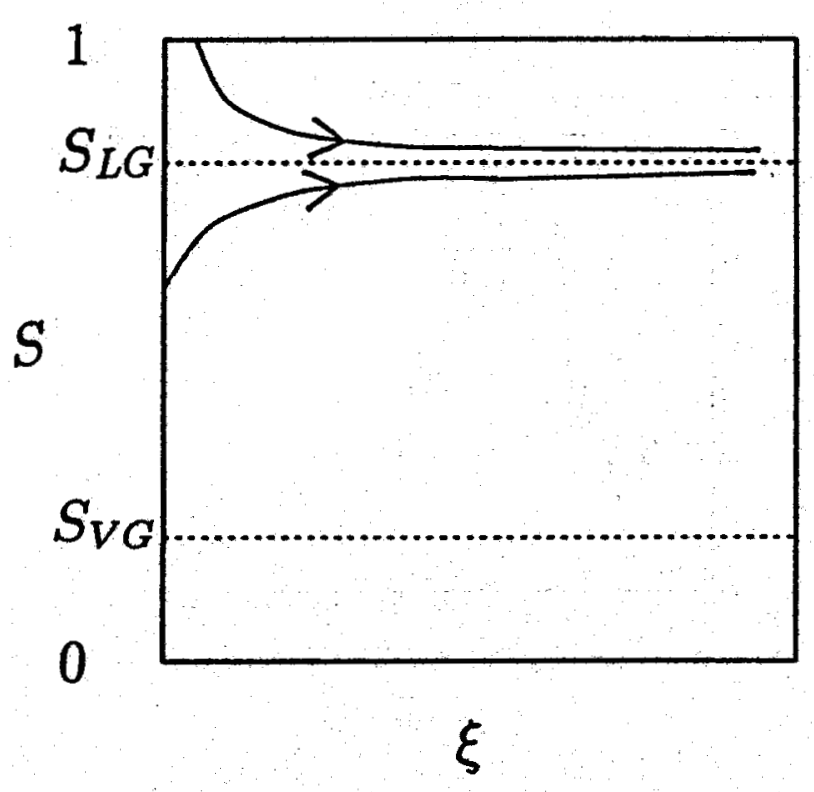

(c)

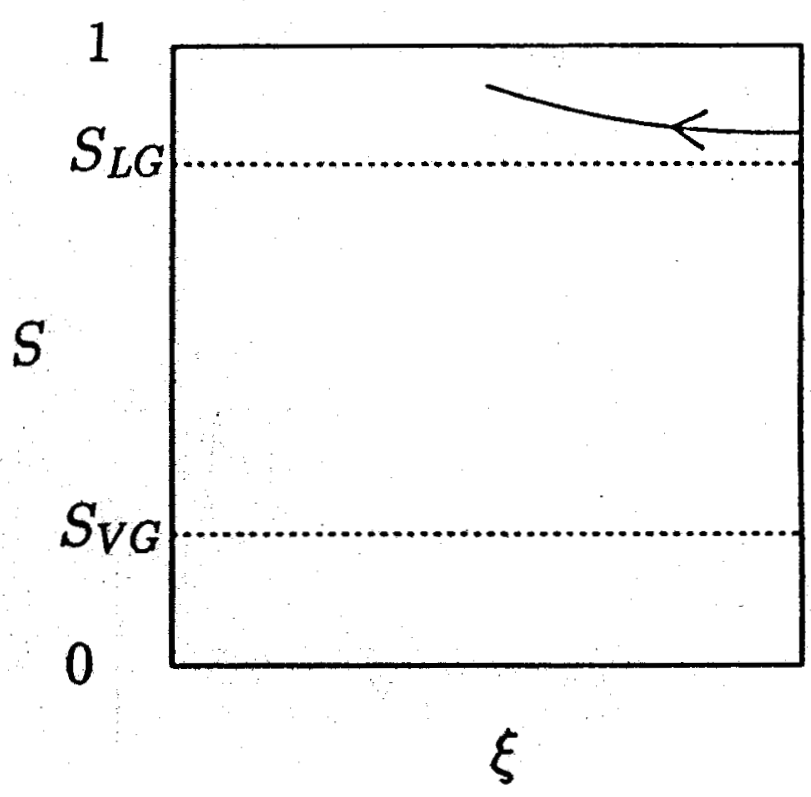

(b)

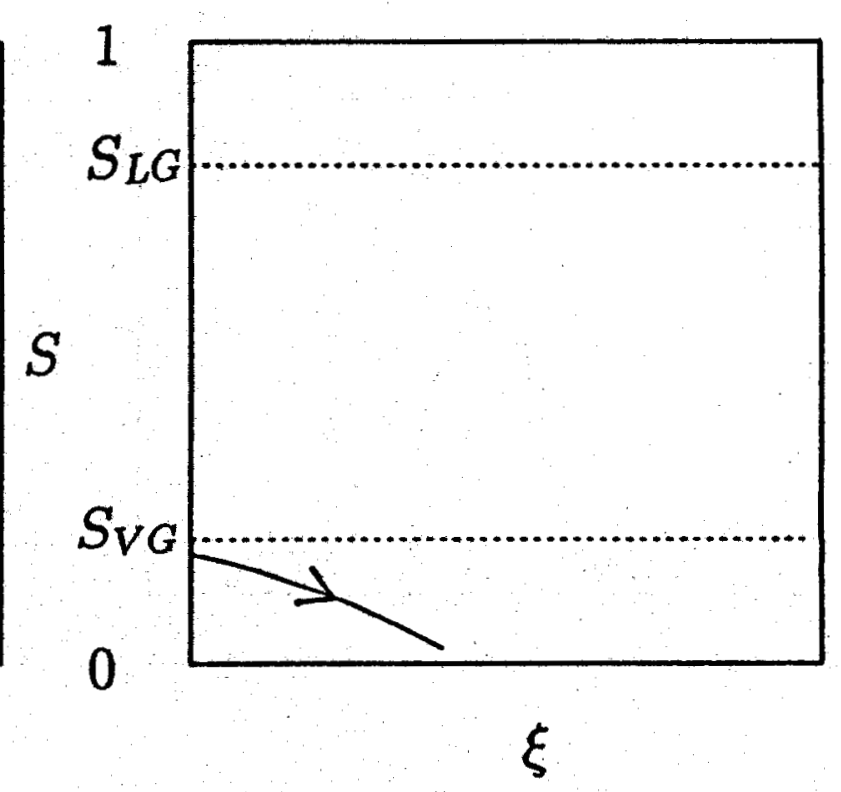

(d)

Figure 5. Steady-state selection in homogeneous heat pipes: (a) $S_{1}<S_{L G}$; (b) $S_{1}>S L G$; (c) $S_{0}>S_{V G}$; (d) $S_{0}<S_{V G}$. Arrow denotes direction of integration. 


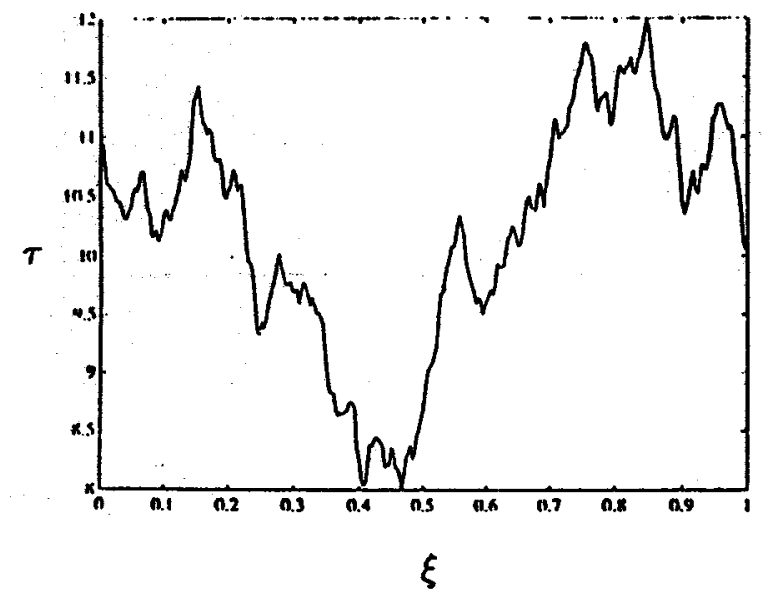

(a)

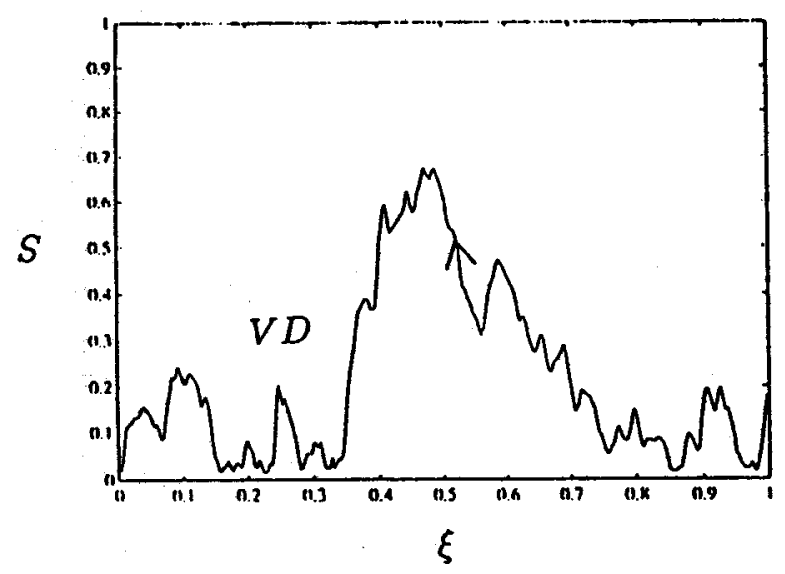

(c)

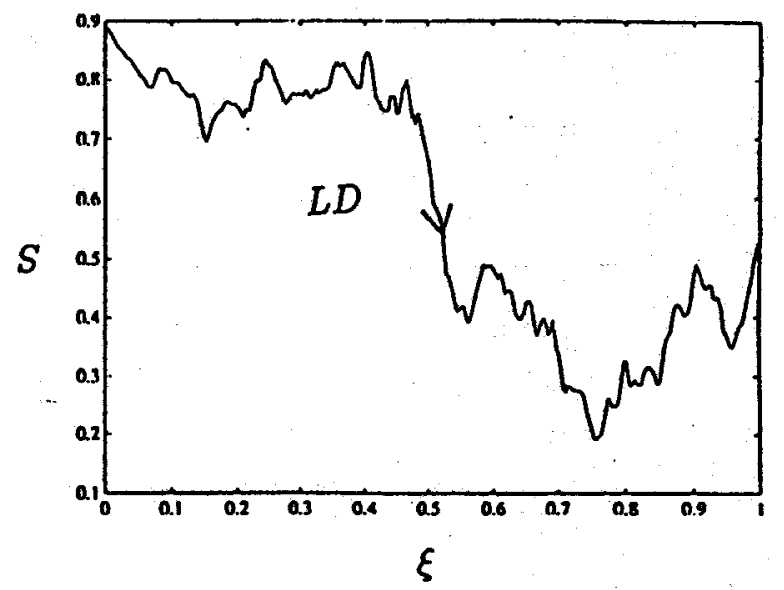

(b)

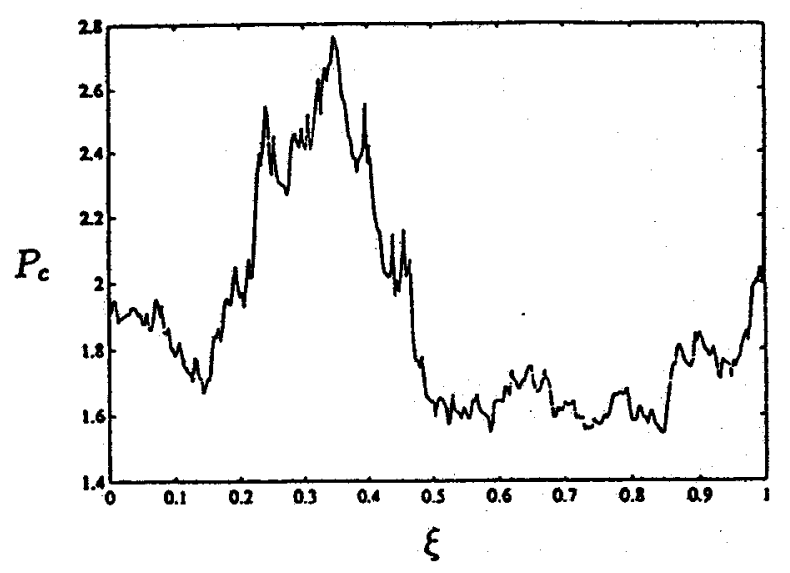

(d)

Figure 6. Normal heterogeneity: (a) $\tau$ profile; (b) $L D$ regime, $\omega=0.02$; (b) $V D$ regime, $\omega=0.02 ;$ (d) dimensionless capillary pressure for the LD regime. 


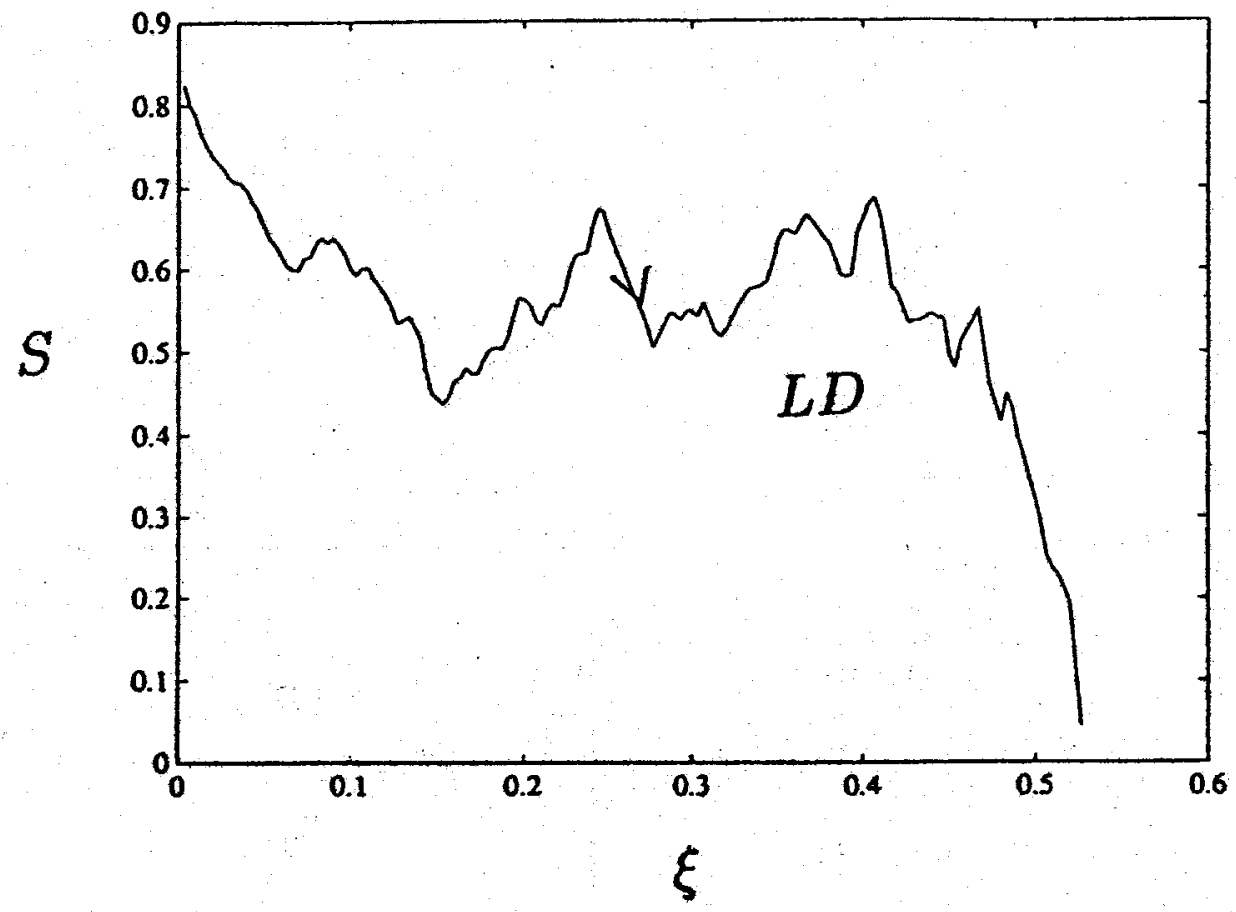

(a)

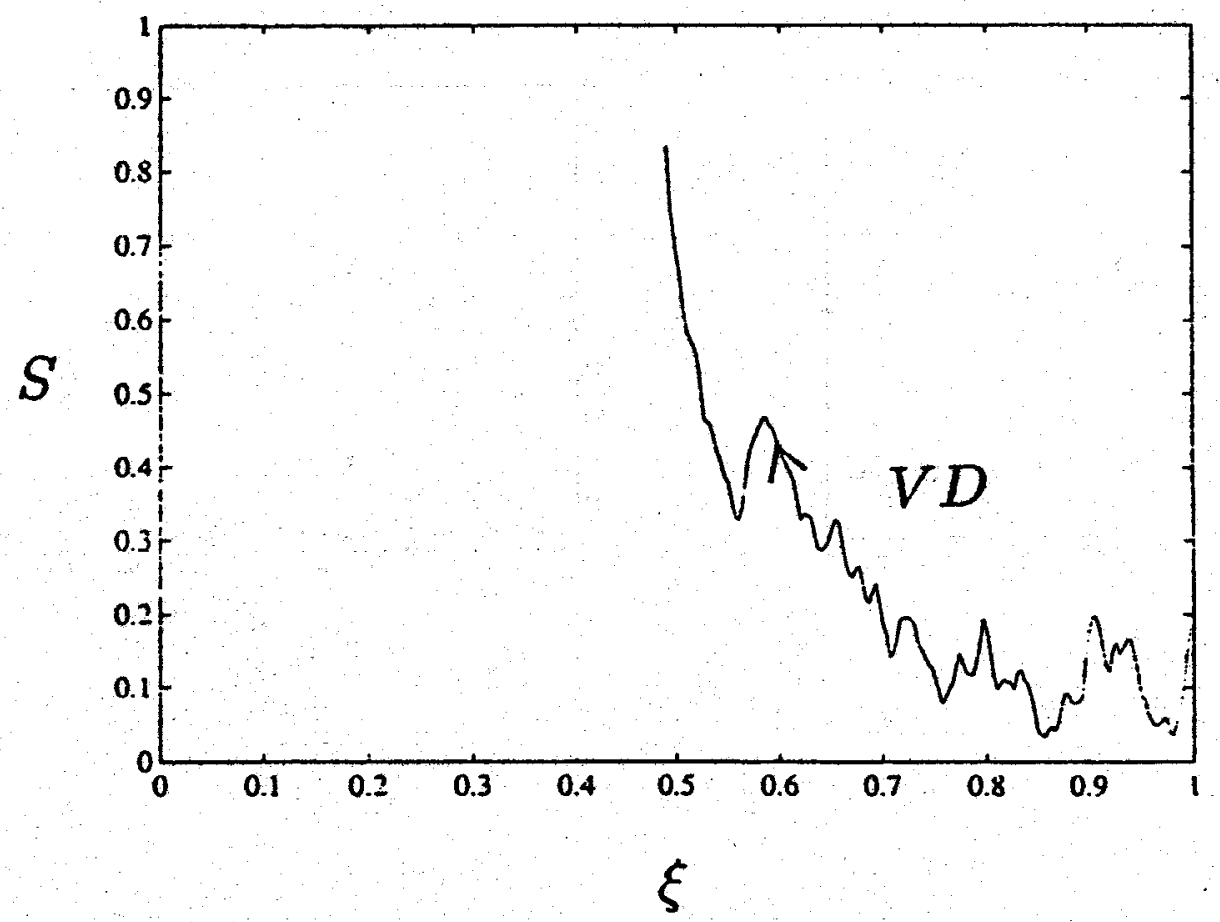

(b)

Figure 7. Transition to single phase flow for larger heat fluxes: (a) $L D$ regime, $\omega=0.2 ;$ (b) $V D$ regime, $\omega=0.2$. 


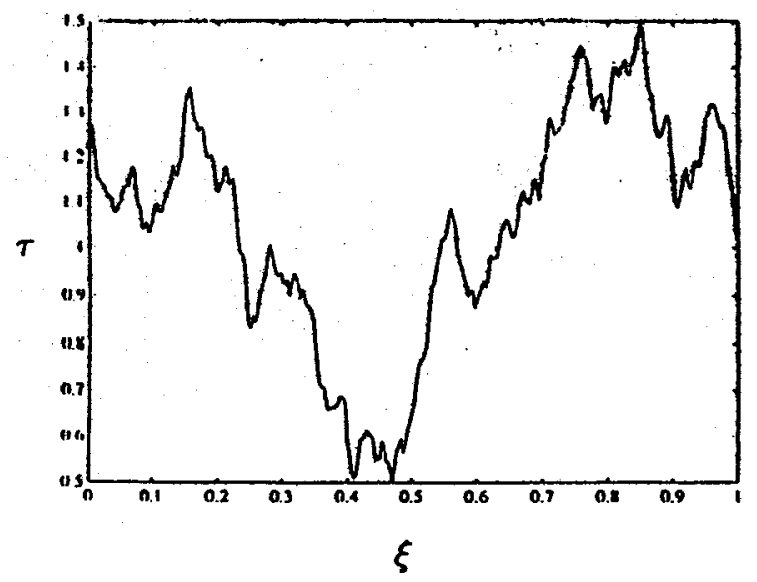

(a)

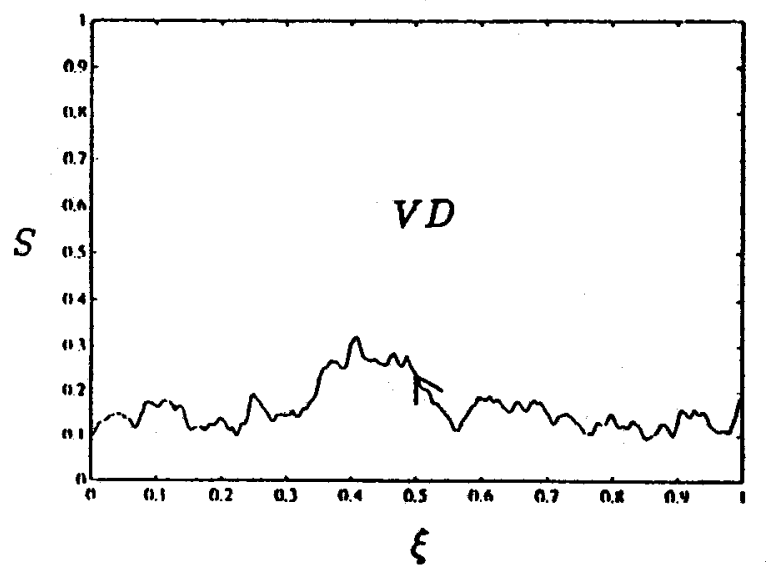

(c)

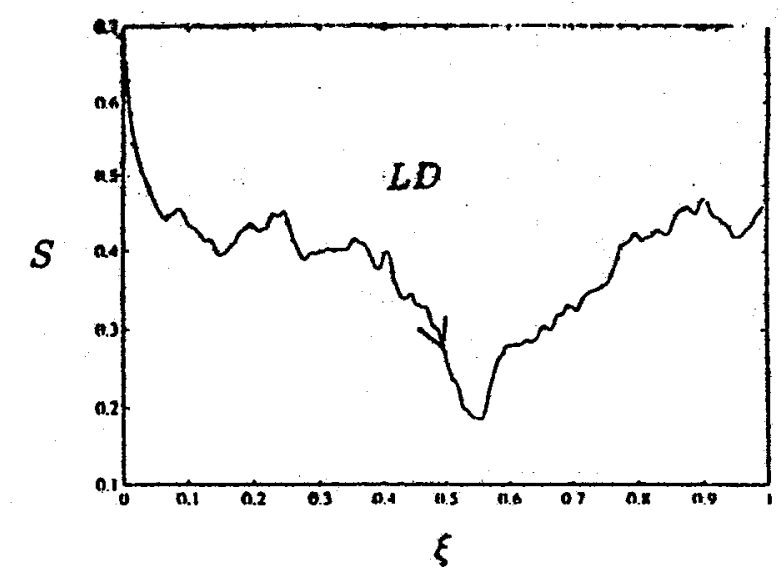

(b)

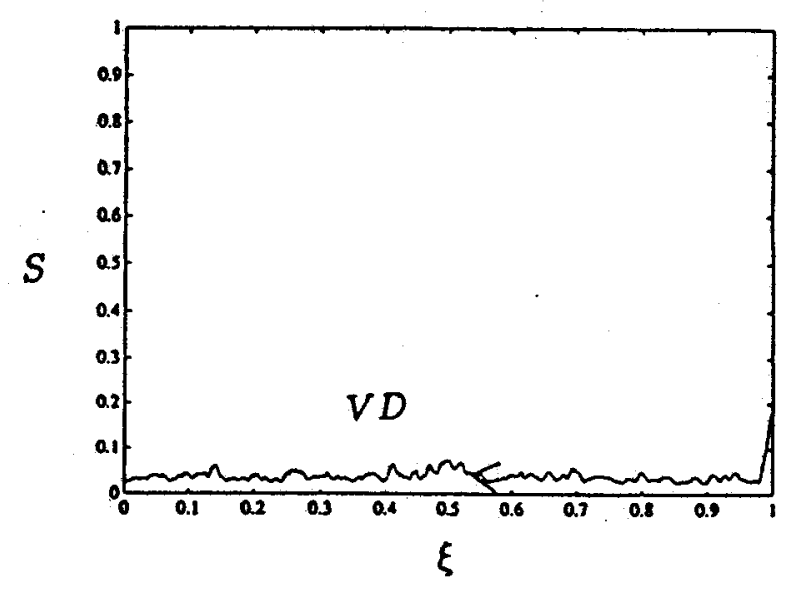

(d)

Figure 8 Weak heterogeneity: (a) $\tau$ profile; (b) $L D$ regime, $\omega=23.0$; (c) $V D$ regime, $\omega=23.0$; (d) $V D$ regime, $\omega=0.6$. 